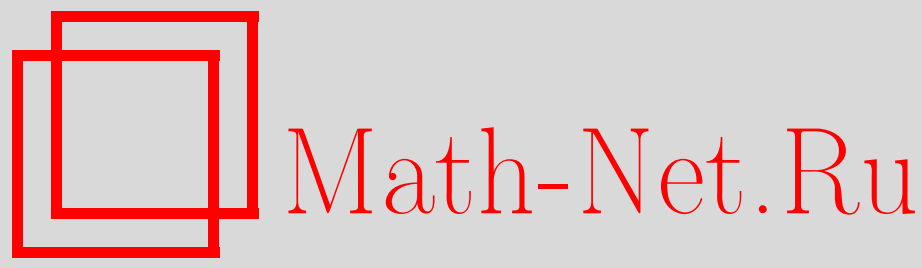

Ф. Коломо, А. Г. Пронько, Подход к вычислению корреляционных функций в шестивершинной модели с граничными условиями доменной стенки, ТМФ, 2012, том 171, номер 2, 254-270

DOI: https://doi.org/10.4213/tmf8368

Использование Общероссийского математического портала Math-Net.Ru подразумевает, что вы прочитали и согласны с пользовательским соглашением http://www.mathnet.ru/rus/agreement

Параметры загрузки:

IP : 3.89.197.203

26 апреля 2023 г., 18:32:38

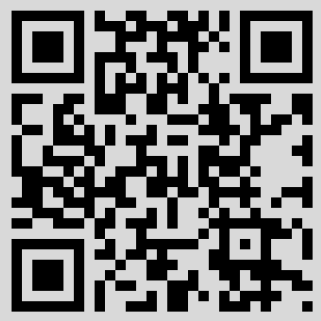




\title{
ПОДХОД К ВЫЧИСЛЕНИЮ КОРРЕЛЯЦИОННЫХ ФУНКЦИЙ В ШЕСТИВЕРШИННОЙ МОДЕЛИ С ГРАНИЧНЫМИ УСЛОВИЯМИ ДОМЕННОЙ СТЕНКИ
}

\begin{abstract}
Задача вычисления корреляционных функций в шестивершинной модели с граничными условиями доменной стенки изучается с помощью некоторой нелокальной корреляционной функции, именуемой вероятностью конфигурации ряда. Эта корреляционная функция может использоваться как строительный блок для вычисления различных (как локальных, так и нелокальных) корреляционных функций в модели. Вероятность конфигурации ряда вычислена квантовым методом обратной задачи; окончательный результат представлен в терминах многократных интегралов. Обсуждается также связь с вероятностью образования пустоты - другой нелокальной корреляционной функцией, которая была вычислена ранее аналогичными методами.
\end{abstract}

Ключевые слова: вершинные модели, корреляционные функции, граничные условия доменной стенки, представления многократными интегралами, квантовый метод обратной задачи.

\section{1. ВВЕДЕНИЕ}

Одной из наиболее фундаментальных задач в теории интегрируемых моделей является точное вычисление корреляционных функций [1]. В последние годы наблюдается возрастающий интерес (мотивированный различными математическими и физическими приложениями) к получению точных результатов для корреляционных функций для моделей статистической механики, заданных на конечных решетках и при фиксированных граничных условиях. В силу нарушенной трансляционной инвариантности систематическое вычисление корреляционных функций для этих моделей представляет собой сложную задачу.

Важным примером такой модели является шестивершинная модель с граничными условиями доменной стенки [2]. Статистическая сумма этой модели на конечной решетке выражается точно через некоторый определитель [3], [4]. Эта формула, известная как детерминантная формула Изергина-Корепина, оказалась мощным инструментом при доказательстве важных комбинаторных результатов. Современный

${ }^{*}$ INFN, Sezione di Firenze, Firenze, Italy. E-mail: colomo@fi.infn.it

${ }^{\dagger}$ Санкт-Петербургское отделение математического института им. В. А. Стеклова РАН, Санкт-Петербург, Россия. E-mail: agp@pdmi.ras.ru 
интерес к модели главным образом мотивирован присутствием явлений разделения фаз (см. работы [5], [6] и библиографию в них).

Определенный прогресс в вычислении корреляционных функций в шестивершинной модели с граничными условиями доменной стенки был достигнут для случая корреляций вблизи границ [7]-[10]. Примером корреляционной функции, которая может быть вычислена вдали от границы, является так называемая вероятность образования пустоты [11]. Некоторые обобщения рассматривались недавно в работе [12]. Тем не менее проблема систематического вычисления корреляционных функций, особенно для случая корреляций вдали от границ, все еще далека от своего решения.

Для изучения этой проблемы в настоящей статье мы вводим некоторую нелокальную корреляционную функцию, именуемую вероятностью конфигурации ряда. Эта корреляционная функция описывает вероятность обнаружения заданной конфигурации стрелок на всех вертикальных ребрах, расположенных между двумя горизонтальными линиями квадратной решетки. Вероятность конфигурации ряда может быть использована как строительный блок для вычисления других (как локальных, так и нелокальных) корреляционных функций. В частности, она тесно связана с вероятностью образования пустоты.

Вероятность конфигурации ряда, будучи интересной для вычисления других корреляционных функций, представляет также и самостоятельный интерес. Существуют ее аналоги в контексте явлений разделения фаз в димерных моделях [13] и в перечислительной комбинаторике [14].

Для вычисления вероятности конфигурации ряда мы рассматриваем неоднородную версию модели и формулируем ее в рамках квантового метода обратной задачи (KMO3) [15] (для обзора см. также книгу [1]). Мы используем тот факт, что вероятность конфигурации ряда может быть представлена как произведение двух сомножителей. Для вычисления первого множителя используется вспомогательный результат работы [16], а для вычисления второго множителя - техника, развитая в работах [8], [10], [11]. Для однородной модели оба множителя представляются в терминах многократных интегралов.

Чтобы показать, как полученные результаты могут быть использованы для вычисления других корреляционных функций, мы обсуждаем здесь связь с вероятностью образования пустоты. Вычисление основано на взятии некоторых сумм и интегралов и использовании тождеств, содержащих антисимметризацию функций многих переменных.

Статья построена следующим образом. В разделе 2 после напоминания основных сведений о модели мы формулируем рассматриваемую задачу в терминах объектов КМОЗ. Вывод вероятности конфигурации ряда приводится в разделах 3 и 4 . Связь с вероятностью образования пустоты обсуждается в разделе 5.

\section{2. ШЕСТИВЕРШИННАЯ МОДЕЛЬ, ГРАНИЧНЫЕ УСЛОВИЯ ДОМЕННОЙ СТЕНКИ И ВЕРОЯТНОСТЬ КОНФИГУРАЦИИ РЯДА}

Мы рассматриваем шестивершинную модель на квадратной решетке, образованной пересечением $N$ горизонтальных и $N$ вертикальных прямых $((N \times N)$-решетка), со специальными фиксированными граничными условиями, называемыми граничными условиями доменной стенки. Напомним, что шестивершинная модель - это модель, в которой локальными состояниями являются стрелки, направленные вдоль 


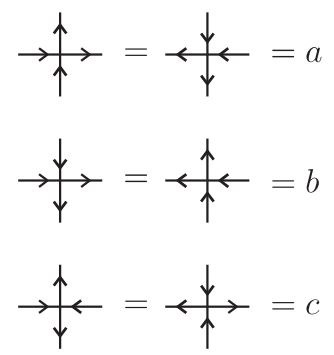

a

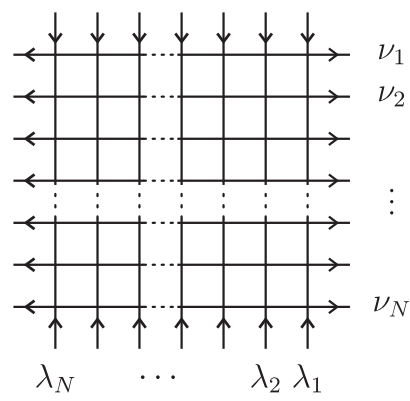

б

Рис. 1. Шесть вершин и их веса (а) и квадратная $(N \times N)$-решетка с граничными условиями доменной стенки (б).

ребер решетки, и разрешенные конфигурации удовлетворяют "правилу льда": каждая вершина должна содержать равное число входящих и исходящих стрелок. Больцмановские веса приписаны шести возможным вершинным конфигурациям стрелок, разрешенных правилом льда; в модели, инвариантной относительно обращения направления всех стрелок, имеются три различных больцмановских веса, обычно обозначаемых как $a, b$ и $c$. Граничные условия доменной стенки означают, что все стрелки на левой и правой границах исходящие, а на верхней и нижней входящие (см. рис. 1).

Чтобы использовать КМОЗ в вычислениях, мы рассматриваем неоднородную версию модели, в которой веса вершины, расположенной на пересечении $\alpha$-й вертикальной прямой (нумеруемой справа) и $k$-й горизонтальной прямой (нумеруемой сверху), равны $a_{\alpha, k}=a\left(\lambda_{\alpha}, \nu_{k}\right), b_{\alpha, k}=b\left(\lambda_{\alpha}, \nu_{k}\right)$ и $c_{\alpha, k}=c$, где

$$
a(\lambda, \nu)=\sin (\lambda-\nu+\eta), \quad b(\lambda, \nu)=\sin (\lambda-\nu-\eta), \quad c=\sin 2 \eta .
$$

Все параметры $\lambda_{1}, \ldots, \lambda_{N}$ предполагаются неравными друг другу; это же предполагается и для $\nu_{1}, \ldots, \nu_{N}$. Параметр

$$
\Delta=\frac{a_{\alpha, k}^{2}+b_{\alpha, k}^{2}-c_{\alpha, k}^{2}}{2 a_{\alpha, k} b_{\alpha, k}}=\cos 2 \eta
$$

принимает одно и то же значение для всех вершин, что гарантирует интегрируемость [17]. Статистическая сумма определяется следующим образом:

$$
Z_{N}=\sum_{C} \prod_{\alpha, k=1}^{N} w_{\alpha, k}(C)
$$

где $w_{\alpha, k}(C)$ принимает значения $w_{\alpha, k}(C)=a_{\alpha k}, b_{\alpha k}, c_{\alpha k}$ в зависимости от конфигурации $C$. Очевидно, что $Z_{N}=Z_{N}\left(\lambda_{1}, \ldots, \lambda_{N} ; \nu_{1}, \ldots, \nu_{N}\right)$, где $\lambda_{1}, \ldots, \lambda_{N}$ и $\nu_{1}, \ldots, \nu_{N}$ могут рассматриваться как "переменные"; параметр $\eta$ имеет смысл "константы связи" и часто опускается в обозначениях. После вычислений с помощью КМОЗ величи́ны для однородной модели (например, статистическая сумма) могут быть получены из таковых для неоднородной модели, если положить $\lambda_{\alpha}=\lambda, \alpha=1, \ldots, N$, 
и $\nu_{k}=\nu, k=1, \ldots, N$, где без потери общности можно также считать $\nu=0$ (см. формулу (2.1)). Мы будем упоминать эту процедуру как однородный предел.

Определим теперь основные объекты КМО3, относящиеся к модели. Во-первых, рассмотрим векторное пространство $\mathbb{C}^{2}$ и обозначим его базисные векторы как состояния "спин-вверх" и "спин-вниз":

$$
|\uparrow\rangle=\left(\begin{array}{l}
1 \\
0
\end{array}\right), \quad|\downarrow\rangle=\left(\begin{array}{l}
0 \\
1
\end{array}\right) .
$$

Каждой горизонтальной и вертикальной прямой решетки сопоставим векторное пространство $\mathbb{C}^{2}$. Мы также будем использовать соглашение, что стрелки вверх и вправо соответствуют состоянию "спин-вверх", а стрелки вниз и влево - состоянию "спин-вниз".

Далее, каждой вершине, лежащей на пересечении $\alpha$-й вертикальной и $k$-й горизонтальной прямых, сопоставим оператор $L_{\alpha, k}\left(\lambda_{\alpha}, \nu_{k}\right)$, который действует нетривиально в прямом произведении двух векторных пространств $\mathbb{C}^{2}$, а именно в "горизонтальном" пространстве $\mathcal{H}_{k}=\mathbb{C}^{2}$ (сопоставленном $k$-й горизонтальной прямой) и "вертикальном" пространстве $\mathcal{V}_{\alpha}=\mathbb{C}^{2}$ (сопоставленном $\alpha$-й вертикальной прямой). Пользуясь аналогией с матрицей рассеяния, стрелочные состояния на верхнем и правом ребрах вершины могут рассматриваться как входящие индексы $L$-оператора, а таковые на нижнем и левом ребрах - как исходящие, что дает

$$
L_{\alpha, k}\left(\lambda_{\alpha}, \nu_{k}\right)=a_{\alpha, k} \frac{1+\tau_{\alpha}^{z} \sigma_{k}^{z}}{2}+b_{\alpha, k} \frac{1-\tau_{\alpha}^{z} \sigma_{k}^{z}}{2}+c_{\alpha, k}\left(\tau_{\alpha}^{-} \sigma_{k}^{+}+\tau_{\alpha}^{+} \sigma_{k}^{-}\right) .
$$

Здесь $\tau_{\alpha}^{l}$ и $\sigma_{k}^{l}, l=+,-, z$, обозначают операторы, действующие как матрицы Паули в $\mathcal{V}_{\alpha}$ и $\mathcal{H}_{k}$ соответственно и тождественно в остальных пространствах.

Упорядоченное произведение $L$-операторов вдоль вертикальной (или горизонтальной) прямой решетки соответствует в КМОЗ матрице монодромии. Чтобы построить, например, матрицу монодромии вдоль вертикальной прямой, полезно рассматривать $L$-оператор как $(2 \times 2)$-матрицу, действующую в пространстве $\mathcal{V}_{\alpha}$, с операторными матричными элементами, действующими в пространстве $\mathcal{H}_{1, \ldots, N}=$ $\bigotimes_{k=1}^{N} \mathcal{H}_{k}$, а именно

$$
L_{\alpha, k}(\lambda, \nu)=\left(\begin{array}{cc}
a(\lambda, \nu) \frac{1+\sigma_{k}^{z}}{2}+b(\lambda, \nu) \frac{1-\sigma_{k}^{z}}{2} & c \cdot \sigma_{k}^{-} \\
c \cdot \sigma_{k}^{+} & b(\lambda, \nu) \frac{1+\sigma_{k}^{z}}{2}+a(\lambda, \nu) \frac{1-\sigma_{k}^{z}}{2}
\end{array}\right)_{\left[\mathcal{V}_{\alpha}\right]}
$$

Здесь нижний индекс в скобках указывает, что это матрица в $\mathcal{V}_{\alpha}$. Упорядоченное произведение вдоль $\alpha$-й вертикальной прямой дает “вертикальную” матрицу монодромии:

$$
\begin{aligned}
T_{\alpha}^{\mathrm{V}}\left(\lambda_{\alpha}\right) & =L_{\alpha, N}\left(\lambda_{\alpha}, \nu_{N}\right) \cdots L_{\alpha, 2}\left(\lambda_{\alpha}, \nu_{2}\right) L_{\alpha, 1}\left(\lambda_{\alpha}, \nu_{1}\right)= \\
& =\left(\begin{array}{ll}
A_{1, \ldots, N}^{\mathrm{V}}\left(\lambda_{\alpha}\right) & B_{1, \ldots, N}^{\mathrm{V}}\left(\lambda_{\alpha}\right) \\
C_{1, \ldots, N}^{\mathrm{V}}\left(\lambda_{\alpha}\right) & D_{1, \ldots, N}^{\mathrm{V}}\left(\lambda_{\alpha}\right)
\end{array}\right)_{\left[\mathcal{V}_{\alpha}\right]} \cdot
\end{aligned}
$$

Операторы $A_{1, \ldots, N}^{\mathrm{V}}(\lambda)=A_{1, \ldots, N}^{\mathrm{V}}\left(\lambda ; \nu_{1}, \ldots, \nu_{N}\right)$ и т. д. действуют в $\mathcal{H}_{1, \ldots, N}$, и они не зависят от $\alpha$. Каждый из этих операторов соответствует вертикальной прямой решетки с фиксированными направлениями самой верхней и самой нижней вертикальных стрелок. 
Аналогично можно рассмотреть “горизонтальную” матрицу монодромии

$$
\begin{aligned}
T_{k}^{\mathrm{H}}\left(\nu_{k}\right) & =L_{N, k}\left(\lambda_{N}, \nu_{k}\right) \cdots L_{2, k}\left(\lambda_{2}, \nu_{k}\right) L_{1, k}\left(\lambda_{1}, \nu_{k}\right)= \\
& =\left(\begin{array}{ll}
A_{1, \ldots, N}^{\mathrm{H}}\left(\nu_{k}\right) & B_{1, \ldots, N}^{\mathrm{H}}\left(\nu_{k}\right) \\
C_{1, \ldots, N}^{\mathrm{H}}\left(\nu_{k}\right) & D_{1, \ldots, N}^{\mathrm{H}}\left(\nu_{k}\right)
\end{array}\right)_{\left[\mathcal{H}_{k}\right]},
\end{aligned}
$$

где операторы $A_{1, \ldots, N}^{\mathrm{H}}(\nu)=A_{1, \ldots, N}^{\mathrm{H}}\left(\nu ; \lambda_{1}, \ldots, \lambda_{N}\right)$ и т. д. действуют в пространстве $\mathcal{V}_{1, \ldots, N}:=\bigotimes_{\alpha=1}^{N} \mathcal{V}_{\alpha}$. Каждый из этих операторов соответствует горизонтальной прямой решетки с фиксированными направлениями самой правой и самой левой горизонтальных стрелок.

Значимость матричных элементов матрицы монодромии заключается в том, что они удовлетворяют квадратичной алгебре, называемой алгеброй матрицы монодромии, или алгеброй Янга-Бакстера [1]. Алгебра в совокупности содержит 16 коммутационных соотношений, и в дальнейшем нам понадобятся некоторые из них, а именно

$$
B(\lambda) B\left(\lambda^{\prime}\right)=B\left(\lambda^{\prime}\right) B(\lambda), \quad C(\lambda) C\left(\lambda^{\prime}\right)=C\left(\lambda^{\prime}\right) C(\lambda)
$$

и

$$
A(\lambda) B\left(\lambda^{\prime}\right)=f\left(\lambda, \lambda^{\prime}\right) B\left(\lambda^{\prime}\right) A(\lambda)+g\left(\lambda^{\prime}, \lambda\right) B(\lambda) A\left(\lambda^{\prime}\right),
$$

где $A(\lambda)=A_{1, \ldots, N}^{\mathrm{V}}(\lambda)$ и т. д., а функции $f\left(\lambda^{\prime}, \lambda\right)$ и $g\left(\lambda^{\prime}, \lambda\right)$ равны

$$
f\left(\lambda^{\prime}, \lambda\right)=\frac{\sin \left(\lambda-\lambda^{\prime}+2 \eta\right)}{\sin \left(\lambda-\lambda^{\prime}\right)}, \quad g\left(\lambda^{\prime}, \lambda\right)=\frac{\sin 2 \eta}{\sin \left(\lambda-\lambda^{\prime}\right)} .
$$

В точности эти же соотношения верны также для $A(\nu)=A_{1, \ldots, N}^{\mathrm{H}}(\nu)$ и т. д. (после замены $\lambda \mapsto \nu$ и $\lambda^{\prime} \mapsto \nu^{\prime}$ в формулах (2.9) и (2.10)). За списком всех коммутационных соотношений, а также описанием их вывода мы отсылаем к главе VIII книги [1].

Теперь мы готовы сформулировать модель в рамках KMO3. Обозначая $|\uparrow V\rangle$ $\left|\downarrow_{k}^{\mathrm{V}}\right\rangle$ базисные векторы пространства $\mathcal{H}_{k}$, введем состояния

$$
\left|\uparrow_{1, \ldots, N}^{\mathrm{V}}\right\rangle:=\bigotimes_{k=1}^{N}\left|\uparrow_{k}^{\mathrm{V}}\right\rangle, \quad\left|\Downarrow_{1, \ldots, N}^{\mathrm{V}}\right\rangle:=\bigotimes_{k=1}^{N}\left|\downarrow_{k}^{\mathrm{V}}\right\rangle
$$

Эти состояния являются состояниями "все спины вверх" и "все спины вниз" соответственно в пространстве $\mathcal{H}_{1, \ldots, N}$. Принимая во внимание, что граничные условия доменной стенки сопоставляют $\alpha$-й вертикальной прямой оператор $B_{1, \ldots, N}^{\mathrm{V}}\left(\lambda_{\alpha}\right)$, мы можем записать статистическую сумму как матричный элемент:

$$
Z_{N}\left(\lambda_{1}, \ldots, \lambda_{N} ; \nu_{1}, \ldots, \nu_{N}\right)=\left\langle\Downarrow_{1, \ldots, N}^{\mathrm{V}}\left|\prod_{\alpha=1}^{N} B_{1, \ldots, N}^{\mathrm{V}}\left(\lambda_{\alpha}\right)\right| \Uparrow_{1, \ldots, N}^{\mathrm{V}}\right\rangle
$$

Напомним также, что $B_{1, \ldots, N}^{\mathrm{V}}(\lambda)=B_{1, \ldots, N}^{\mathrm{V}}\left(\lambda ; \nu_{1}, \ldots, \nu_{N}\right)$.

Аналогично статистическая сумма может быть построена с помощью операторов, ассоциированных с горизонтальными прямыми. Обозначая через $\left|\uparrow_{\alpha}^{\mathrm{H}}\right\rangle$ и $\left|\downarrow_{\alpha}^{\mathrm{H}}\right\rangle$ базисные векторы пространства $\mathcal{V}_{\alpha}$, мы можем ввести состояния

$$
\left|\uparrow_{1, \ldots, N}^{\mathrm{H}}\right\rangle:=\bigotimes_{\alpha=1}^{N}\left|\uparrow_{\alpha}^{\mathrm{H}}\right\rangle, \quad\left|\Downarrow_{1, \ldots, N}^{\mathrm{H}}\right\rangle:=\bigotimes_{\alpha=1}^{N}\left|\downarrow_{\alpha}^{\mathrm{H}}\right\rangle,
$$




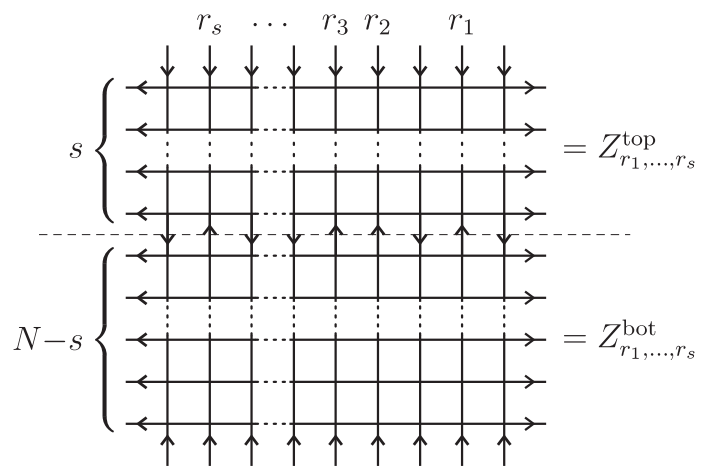

Рис. 2. Конфигурация ряда с $s$ стрелками, направленными вверх, лежащими между $s$-й и $(s+1)$-й горизонтальными прямыми сверху, и результирующие статистические суммы верхней и нижней частей оригинальной решетки.

которые являются состояниями “все спины вверх" и “все спины вниз" пространства $\mathcal{V}_{1, \ldots, N}$. Статистическая сумма равна

$$
Z_{N}\left(\lambda_{1}, \ldots, \lambda_{N} ; \nu_{1}, \ldots, \nu_{N}\right)=\left\langle\Uparrow_{1, \ldots, N}^{\mathrm{H}}\left|\prod_{k=1}^{N} C_{1, \ldots, N}^{\mathrm{H}}\left(\nu_{k}\right)\right| \Downarrow_{1, \ldots, N}^{\mathrm{H}}\right\rangle
$$

и мы напомним, что $C_{1, \ldots, N}^{\mathrm{H}}(\nu)=C_{1, \ldots, N}^{\mathrm{H}}\left(\nu ; \lambda_{1}, \ldots, \lambda_{N}\right)$.

Известно, что статистическая сумма выражается детерминантной формулой Изергина-Корепина (см. работы [2]-[4]):

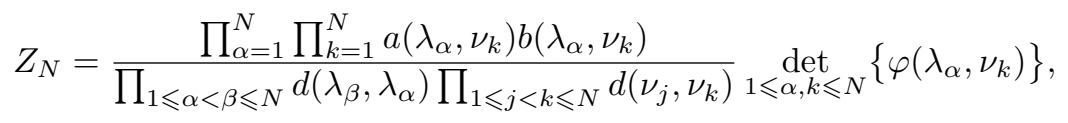

где $d\left(\lambda, \lambda^{\prime}\right):=\sin \left(\lambda-\lambda^{\prime}\right)$ и

$$
\varphi(\lambda, \nu)=\frac{c}{a(\lambda, \nu) b(\lambda, \nu)}
$$

а $a(\lambda, \nu), b(\lambda, \nu)$ и $c$ определены формулой (2.1). Оригинальное доказательство формулы (2.16) содержится в работе [4]; альтернативный вывод этой формулы может быть найден в работах [8], [11].

В однородном пределе, т. е. когда $\lambda_{1}=\cdots=\lambda_{N}=\lambda$ и $\nu_{1}=\cdots=\nu_{N}=0$, выражение (2.16) приобретает вид

$$
Z_{N}(\lambda, \ldots, \lambda ; 0, \ldots, 0)=\frac{[\sin (\lambda-\eta) \sin (\lambda+\eta)]^{N^{2}}}{\prod_{n=1}^{N-1}(n !)^{2}} \operatorname{det}_{1 \leqslant \alpha, k \leqslant N}\left\{\partial_{\lambda}^{\alpha+k-2} \varphi(\lambda)\right\}
$$

где $\varphi(\lambda):=\varphi(\lambda, 0)$. Ниже мы часто используем упрощенные обозначения для величин однородной модели, например, $Z_{N}$ для $Z_{N}(\lambda, \ldots, \lambda ; 0, \ldots, 0)$ и т. д. 
Обратимся теперь к вероятности конфигурации ряда. Чтобы определить эту величину, полезно отметить вначале, что в шестивершинной модели с граничными условиями доменной стенки все конфигурации таковы, что на $s$-м ряду (т. е. на $N$ вертикальных ребрах, расположенных между $s$-й и $(s+1)$-й горизонтальными прямыми, нумеруемыми сверху по нашим соглашениям) ровно $s$ стрелок направлены вверх. Таким образом, естественно исследовать вероятность нахождения заданной конфигурации стрелок на заданном ряду, или, кратко, вероятность конфигурации ряда. А именно, мы обозначим через $H_{N, s}^{\left(r_{1}, \ldots, r_{s}\right)}$ вероятность того, что $s$ стрелок, направленных вверх, $s$-го ряда находятся в точности в позициях $r_{1}, \ldots, r_{s}$ (нумеруемых справа) (см. рис. 2).

Поскольку вероятность конфигурации ряда описывает типичные конфигурации модели, эта величина может быть использована как строительный блок для вычисления других корреляционных функций. Например, суммируя подходящим образом по позициям стрелок, направленных вверх, можно воспроизвести так называемую вероятность образования пустоты, которая исследовалась в работе [11]. Эта связь обсуждается в разделе 5 .

Чтобы вычислить вероятность конфигурации ряда, разделим начальную $(N \times N)$ решетку на две меньшие: верхнюю решетку с $s$ горизонтальными и $N$ вертикальными прямыми и нижнюю решетку с $N-s$ горизонтальными и $N$ вертикальными прямыми. Будем обозначать через $Z_{r_{1}, \ldots, r_{s}}^{\mathrm{top}}$ и $Z_{r_{1}, \ldots, r_{s}}^{\mathrm{bot}}$ статистические суммы шестивершинной модели на верхней и нижней подрешетках соответственно (см. рис. 2). Вероятность конфигурации ряда дается как произведение статистических сумм шестивершинной модели на этих двух меньших решетках:

$$
H_{N, s}^{\left(r_{1}, \ldots, r_{s}\right)}=\frac{Z_{r_{1}, \ldots, r_{s}}^{\mathrm{top}} Z_{r_{1}, \ldots, r_{s}}^{\mathrm{bot}}}{Z_{N}} .
$$

Таким образом, нашей основной задачей в настоящей работе является вывод некоторых полезных представлений для статистических сумм $Z_{r_{1}, \ldots, r_{s}}^{\text {top }} Z_{r_{1}, \ldots, r_{s}}^{\text {bot }}$. Точнее, мы предложим представления через многократные ( $s$-кратные) интегралы для этих величин.

В терминах объектов КМОЗ статистическая сумма на верхней $(s \times N)$-подрешетке может быть записана аналогично представлению (2.15) следующим образом:

$$
Z_{r_{1}, \ldots, r_{s}}^{\mathrm{top}}=\left\langle\Downarrow_{1, \ldots, N}^{\mathrm{H}}\left|\tau_{r_{s}}^{-} \cdots \tau_{r_{2}}^{-} \tau_{r_{1}}^{-} \prod_{k=1}^{s} C_{1, \ldots, N}^{\mathrm{H}}\left(\nu_{k}\right)\right| \Downarrow_{1, \ldots, N}^{\mathrm{H}}\right\rangle,
$$

где $\tau_{j}^{-}, j=1, \ldots, N$, как и выше, обозначает матрицы Паули, действующие в пространствах $\mathcal{V}_{j}$.

Для того чтобы записать статистическую сумму нижней $((N-s) \times N)$-подрешетки как матричный элемент, определим векторы

$$
\left|\Uparrow_{s+1, \ldots, N}^{\mathrm{V}}\right\rangle=\bigotimes_{k=s+1}^{N}\left|\uparrow_{k}^{\mathrm{V}}\right\rangle, \quad\left|\Downarrow_{s+1, \ldots, N}^{\mathrm{V}}\right\rangle=\bigotimes_{k=s+1}^{N}\left|\downarrow_{k}^{\mathrm{V}}\right\rangle
$$


Эти векторы являются состояниями "все спины вверх" и “все спины вниз" пространства $\mathcal{H}_{s+1, \ldots, N}$. Соответственно рассмотрим матричные элементы "укороченной" вертикальной матрицы монодромии, определенной в виде произведения $L_{\alpha, N}\left(\lambda_{\alpha}, \nu_{N}\right) \cdots L_{\alpha, s+1}\left(\lambda_{\alpha}, \nu_{s+1}\right)$. Эти матричные элементы являются операторами $A_{s+1, \ldots, N}^{\mathrm{V}}\left(\lambda_{\alpha}\right)=A_{s+1, \ldots, N}^{\mathrm{V}}\left(\lambda_{\alpha} ; \nu_{s+1}, \ldots, \nu_{N}\right)$ и т. д., действующими в пространстве $\mathcal{H}_{s+1, \ldots, N}$. Статистическая сумма $Z_{r_{1}, \ldots, r_{s}}^{\text {bot }}$ может быть записана как

$$
\begin{aligned}
Z_{r_{1}, \ldots, r_{s}}^{\mathrm{bot}}=\left\langle\Downarrow_{s+1, \ldots, N}^{\mathrm{V}}\right| \prod_{\alpha=r_{s}+1}^{N} B\left(\lambda_{\alpha}\right) \cdot A\left(\lambda_{r_{s}}\right) \cdot \prod_{\alpha=r_{s-1}+1}^{r_{s}-1} B\left(\lambda_{\alpha}\right) \times \cdots \\
\\
\quad \cdots \times A\left(\lambda_{r_{2}}\right) \prod_{\alpha=r_{1}+1}^{r_{2}-1} B\left(\lambda_{\alpha}\right) \cdot A\left(\lambda_{r_{1}}\right) \cdot \prod_{\alpha=1}^{r_{1}-1} B\left(\lambda_{\alpha}\right)\left|\Uparrow_{s+1, \ldots, N}^{\mathrm{V}}\right\rangle,
\end{aligned}
$$

где $A(\lambda):=A_{s+1, \ldots, N}^{\mathrm{V}}(\lambda)$ и $B(\lambda):=B_{s+1, \ldots, N}^{\mathrm{V}}(\lambda)$.

\section{3. ВЫЧИСЛЕНИЕ $Z_{r_{1}, \ldots, r_{s}}^{\mathrm{top}}$}

Матричный элемент в выражении (2.20) может быть формально вычислен (как функция $\lambda_{1}, \ldots, \lambda_{N}$ и $\left.\nu_{1}, \ldots, \nu_{s}\right)$ с использованием эквивалентности алгебраической и координатной версий анзаца Бете. Эта эквивалентность была впервые доказана в явном виде как вспомогательный результат в работе [16] (см. приложение D работы [16], а также главу VII книги [1]).

Для простоты мы начнем сразу со случая, когда параметры $\lambda_{1}, \ldots, \lambda_{N}$ приравнены к одному и тому же значению $\lambda$, однако параметры $\nu_{1}, \ldots, \nu_{s}$ остаются произвольными (и не равными друг другу). Формула (D.4) работы [16] в этом случае означает

$$
\begin{aligned}
Z_{r_{1}, \ldots, r_{s}}^{\mathrm{top}}= & c^{s} \prod_{k=1}^{s}\left[a\left(\lambda, \nu_{k}\right)\right]^{N-1} \prod_{1 \leqslant j<k \leqslant s} \frac{1}{t_{k}-t_{j}} \times \\
& \times \sum_{P \in \Omega_{s}}(-1)^{[P]} \prod_{j=1}^{s} t_{P_{j}}^{r_{j}-1} \prod_{1 \leqslant j<k \leqslant s}\left(t_{P_{j}} t_{P_{k}}-2 \Delta t_{P_{j}}+1\right),
\end{aligned}
$$

где

$$
t_{k}:=\frac{b\left(\lambda, \nu_{k}\right)}{a\left(\lambda, \nu_{k}\right)}
$$

и сумма вычисляется по элементам симметрической группы $\Omega_{s}$, т. е. по перестановкам $P: 1, \ldots, s \mapsto P_{1}, \ldots, P_{s}$, где $[P]$ обозначает четность $P$. Очевидно, что сумма в выражении (3.1) является в точности подстановкой для $s$-частичной волновой функции координатного анзаца Бете.

Для того чтобы исследовать однородный предел выражения (3.1) для остального набора параметров, преобразуем сначала немного это выражение. Положим $t_{k}=t+x_{k}, k=1, \ldots, k$, где $t$ является произвольным параметром и все новые параметры $x_{1}, \ldots, x_{s}$ различны. Используя тот факт, что для функции $f(x)$, регулярной в окрестности точки $x=t$, всегда можно написать $f(t+x)=\left.e^{x \partial_{z}} f(t+z)\right|_{z=0}$, 
приведем выражение (3.1) к виду

$$
\begin{aligned}
Z_{r_{1}, \ldots, r_{s}}^{\mathrm{top}}= & c^{s} \prod_{k=1}^{s}\left[a\left(\lambda, \nu_{k}\right)\right]^{N-1} \prod_{1 \leqslant j<k \leqslant s} \frac{1}{x_{k}-x_{j}} \operatorname{det}_{1 \leqslant j, k \leqslant s}\left\{e^{\left.x_{j} \partial_{z_{k}}\right\} \times}\right. \\
& \times\left.\prod_{j=1}^{s}\left(t+z_{j}\right)^{r_{j}-1} \prod_{1 \leqslant j<k \leqslant s}\left[\left(t+z_{j}\right)\left(t+z_{k}\right)-2 \Delta\left(t+z_{j}\right)+1\right]\right|_{z_{1}=\cdots=z_{s}=0},
\end{aligned}
$$

что представляет собой просто эквивалентный способ записи формулы (3.1).

Рассмотрим теперь однородный предел по параметрам $\nu_{1}, \ldots, \nu_{s}$. Поскольку $t$ произвольно, мы можем выполнить этот предел так, чтобы было верно $t_{1}=\cdots$ $\cdots=t_{k}=t$, и положить $t=b / a$, где $a$ и $b$ являются весами однородной модели (см. формулу (2.1)). Таким образом, мы должны рассмотреть выражение (3.1) при $x_{1}=\cdots=x_{s}=0$. Этот предел в формуле (3.3) может быть вычислен при помощи соотношения

$$
\left.\frac{\operatorname{det}_{1 \leqslant j, k \leqslant s}\left\{e^{x_{j} \partial_{z_{k}}}\right\}}{\prod_{1 \leqslant j<k \leqslant s}\left(x_{k}-x_{j}\right)}\right|_{x_{1}=\cdots=x_{s}=0}=\operatorname{det}_{1 \leqslant j, k \leqslant s}\left\{\frac{1}{(j-1) !} \partial_{z_{k}}^{j-1}\right\} .
$$

Выражая значения производных при $z_{1}=\cdots=z_{s}=0$ как вычеты в полюсах, получим представление многократным интегралом

$$
\begin{aligned}
Z_{r_{1}, \ldots, r_{s}}^{\mathrm{top}}= & c^{s} a^{s(N-1)} \oint_{C_{0}} \cdots \oint_{C_{0}} \prod_{j=1}^{s}\left(t+z_{j}\right)^{r_{j}-1} \operatorname{det}_{1 \leqslant j, k \leqslant s}\left\{z_{k}^{-j}\right\} \times \\
& \times \prod_{1 \leqslant j<k \leqslant s}\left[\left(t+z_{j}\right)\left(t+z_{k}\right)-2 \Delta\left(t+z_{j}\right)+1\right] \frac{d^{s} z}{(2 i \pi)^{s}} .
\end{aligned}
$$

Здесь $C_{0}$ обозначает малый простой замкнутый положительно ориентированный контур, обходящий точку $z=0$. Вычисляя определитель Вандермонда и выполняя замену $z_{k} \mapsto w_{k}=\left(z_{k}+t\right) / t$, окончательно получим

$$
\begin{aligned}
Z_{r_{1}, \ldots, r_{s}}^{\mathrm{top}}= & c^{s} a^{s(N-1)} \prod_{j=1}^{s} t^{r_{j}-j} \oint_{C_{1}} \cdots \oint_{C_{1}} \prod_{j=1}^{s} \frac{w_{j}^{r_{j}-1}}{\left(w_{j}-1\right)^{s}} \times \\
& \times \prod_{1 \leqslant j<k \leqslant s}\left[\left(w_{j}-w_{k}\right)\left(t^{2} w_{j} w_{k}-2 \Delta t w_{j}+1\right)\right] \frac{d^{s} w}{(2 \pi i)^{s}} .
\end{aligned}
$$

Здесь $C_{1}$ обозначает замкнутый контур, обходящий точку $z=1$.

Формулы (3.1) и (3.6) могут быть также выведены другими методами (т. е. без использования эквивалентности алгебраического и координатного анзацев Бете), например, начиная с формулировки в терминах вертикальной матрицы монодромии для $Z_{r_{1}, \ldots, r_{s}}^{\text {top }}$, аналогичной формуле $(2.22)$ для $Z_{r_{1}, \ldots, r_{s}}^{\text {bot }}$, и применяя далее технику работы [18] для вычисления матричного элемента ${ }^{1)}$. Также упомянем, что формула (3.3) в другой форме и для специальных значений $t$ и $\Delta$ была обнаружена в контексте перечислительной комбинаторики [19].

\footnotetext{
1) Мы благодарим П. Зинн-Жюстена за объяснение этого альтернативного вывода.
} 


\section{4. ВЫЧИСЛЕНИЕ $Z_{r_{1}, \ldots, r_{s}}^{\text {bot }}$}

Принимая во внимание перестановочность $B$-операторов (см. формулу $(2.9))$ и используя соотношение (2.10), нетрудно получить в обычном стиле вычислений в алгебраическом анзаце Бете (подробности см., например, в книге [1]) соотношение

$$
A\left(\lambda_{r}\right) \prod_{\beta=1}^{r-1} B\left(\lambda_{\beta}\right)=\sum_{\alpha=1}^{r} \frac{g\left(\lambda_{\alpha}, \lambda_{r}\right)}{f\left(\lambda_{\alpha}, \lambda_{r}\right)} \prod_{\substack{\beta=1 \\ \beta \neq \alpha}}^{r} f\left(\lambda_{\alpha}, \lambda_{\beta}\right) \prod_{\substack{\beta=1 \\ \beta \neq \alpha}}^{r} B\left(\lambda_{\beta}\right) A\left(\lambda_{\alpha}\right) .
$$

Используя это соотношение и принимая во внимание, что

$$
A_{s+1, \ldots, N}^{\mathrm{V}}(\lambda)\left|\Uparrow_{s+1, \ldots, N}^{\mathrm{V}}\right\rangle=\prod_{k=s+1}^{N} a\left(\lambda, \nu_{k}\right)\left|\Uparrow_{s+1, \ldots, N}^{\mathrm{V}}\right\rangle,
$$

а также используя формулу (2.13), мы получим

$$
\begin{aligned}
Z_{r_{1}, \ldots, r_{s}}^{\text {bot }}= & \sum_{\alpha_{1}=1}^{r_{1}} \sum_{\substack{\alpha_{2}=1 \\
\alpha_{2} \neq \alpha_{1}}}^{r_{2}} \ldots \sum_{\substack{\alpha_{s}=1 \\
\alpha_{s} \neq \alpha_{1}, \ldots, \alpha_{s-1}}}^{r_{s}} \prod_{j=1}^{s} \prod_{k=s+1}^{N} a\left(\lambda_{\alpha_{j}}, \nu_{k}\right) \prod_{j=1}^{s} \frac{g\left(\lambda_{\alpha_{j}}, \lambda_{r_{j}}\right)}{f\left(\lambda_{\alpha_{j}}, \lambda_{r_{j}}\right)} \times \\
& \times \prod_{\substack{\beta_{1}=1 \\
\beta_{1} \neq \alpha_{1}}}^{r_{1}} f\left(\lambda_{\alpha_{1}}, \lambda_{\beta_{1}}\right) \prod_{\substack{\beta_{2}=1 \\
\beta_{2} \neq \alpha_{1}, \alpha_{2}}}^{r_{2}} f\left(\lambda_{\alpha_{2}}, \lambda_{\beta_{2}}\right) \cdots \prod_{\substack{\beta_{s}=1 \\
\beta_{s} \neq \alpha_{1}, \ldots, \alpha_{s}}}^{r_{s}} f\left(\lambda_{\alpha_{s}}, \lambda_{\beta_{s}}\right) \times \\
& \times Z_{N-s}\left(\lambda_{\bar{\alpha}_{1}}, \ldots, \lambda_{\bar{\alpha}_{N-s}} ; \nu_{s+1}, \ldots, \nu_{N}\right),
\end{aligned}
$$

где $\left\{\bar{\alpha}_{1}, \ldots, \bar{\alpha}_{N-s}\right\}:=\{1, \ldots, N\} \backslash\left\{\alpha_{1}, \ldots, \alpha_{s}\right\}$.

Для дальнейших вычислений удобно ввести функцию

$$
v_{r}(\lambda)=\frac{\prod_{\alpha=r+1}^{N} d\left(\lambda_{\alpha}, \lambda\right) \prod_{\alpha=1}^{r-1} e\left(\lambda_{\alpha}, \lambda\right)}{\prod_{k=s+1}^{N} b\left(\lambda, \nu_{k}\right)},
$$

где $d\left(\lambda, \lambda^{\prime}\right):=\sin \left(\lambda-\lambda^{\prime}\right)$ и $e\left(\lambda, \lambda^{\prime}\right):=\sin \left(\lambda-\lambda^{\prime}+2 \eta\right)$. Выражая функции $f\left(\lambda, \lambda^{\prime}\right)$ и $g\left(\lambda, \lambda^{\prime}\right)$, фигурирующие в формуле (4.3), в терминах функций $d\left(\lambda, \lambda^{\prime}\right)$ и $e\left(\lambda, \lambda^{\prime}\right)$, и подставляя детерминантную формулу Изергина-Корепина (см. (2.16)) для статистической суммы, стоящей в формуле (4.3), придем к выражению

$$
\begin{aligned}
Z_{r_{1}, \ldots, r_{s}}^{\text {bot }}= & \frac{\prod_{\alpha=1}^{N} \prod_{k=s+1}^{N} a\left(\lambda_{\alpha}, \nu_{k}\right) b\left(\lambda_{\alpha}, \nu_{k}\right)}{\prod_{1 \leqslant \alpha<\beta \leqslant N} d\left(\lambda_{\beta}, \lambda_{\alpha}\right) \prod_{s+1 \leqslant j<k \leqslant N} d\left(\nu_{j}, \nu_{k}\right)} \times \\
& \times \sum_{\alpha_{1}}^{r_{1}} \sum_{\substack{\alpha_{2}=1 \\
\alpha_{2} \neq \alpha_{1}}}^{r_{2}} \ldots \sum_{\substack{\alpha_{s}=1 \\
\alpha_{s} \neq \alpha_{1}, \ldots, \alpha_{s-1}}}^{r_{s}}(-1)^{\sum_{j=1}^{s}\left(\alpha_{j}-1\right)-\sum_{1 \leqslant j<k \leqslant s} \chi\left(\alpha_{k}, \alpha_{j}\right) \times} \\
& \times \prod_{j=1}^{s} v_{r_{j}}\left(\lambda_{\alpha_{j}}\right) \prod_{1 \leqslant j<k \leqslant s} \frac{1}{e\left(\lambda_{\alpha_{j}}, \lambda_{\alpha_{k}}\right)} \underset{1 \leqslant j, k \leqslant N-s}{\operatorname{det}_{1 \leqslant j}\left\{\varphi\left(\lambda_{\bar{\alpha}_{j}}, \nu_{s+k}\right)\right\} .}
\end{aligned}
$$

Здесь $\chi(\beta, \alpha)=1$, если $\beta>\alpha$, и $\chi(\beta, \alpha)=0$ в противном случае.

Очевидно, что многократная сумма в выражении (4.5) напоминает разложение Лапласа некоторого $(N \times N)$-определителя. Это также согласуется с тем фактом, 
что, поскольку $v_{r}\left(\lambda_{\alpha}\right)=0(\alpha=r+1, \ldots, N)$, все суммирования в формуле $(4.5)$ могут быть распространены вплоть до значения $N$. Для того чтобы получить эту детерминантную формулу, положим $\lambda_{\alpha}=\lambda+\xi_{\alpha}, \alpha=1, \ldots, N$, где $\lambda$ - некоторый произвольный параметр и все $\xi_{1}, \ldots, \xi_{N}$ различны. Вновь используя тот факт, что для функции $f(\xi)$, регулярной в окрестности точки $\xi=\lambda$, можно написать $f(\lambda+\xi)=$ $\left.e^{\xi \partial_{\varepsilon}} f(\lambda+\varepsilon)\right|_{\varepsilon=0}$, приведем выражение (4.5) к виду

$$
\begin{aligned}
& Z_{r_{1}, \ldots, r_{s}}^{\text {bot }}=\frac{\prod_{\alpha=1}^{N} \prod_{k=s+1}^{N} a\left(\lambda_{\alpha}, \nu_{k}\right) b\left(\lambda_{\alpha}, \nu_{k}\right)}{\prod_{1 \leqslant \alpha<\beta \leqslant N} d\left(\lambda_{\beta}, \lambda_{\alpha}\right) \prod_{s+1 \leqslant j<k \leqslant N} d\left(\nu_{j}, \nu_{k}\right)} \times \\
& \times\left|\begin{array}{cccccc}
e^{\xi_{1} \partial_{\varepsilon_{1}}} & \cdots & e^{\xi_{1} \partial_{\varepsilon_{s}}} & \varphi\left(\lambda_{1}, \nu_{s+1}\right) & \cdots & \varphi\left(\lambda_{1}, \nu_{N}\right) \\
e^{\xi_{2} \partial_{\varepsilon_{1}}} & \cdots & e^{\xi_{2} \partial_{\varepsilon_{s}}} & \varphi\left(\lambda_{2}, \nu_{s+1}\right) & \cdots & \varphi\left(\lambda_{2}, \nu_{N}\right) \\
\vdots & \ddots & \vdots & \vdots & \ddots & \vdots \\
e^{\xi_{N} \partial_{\varepsilon_{1}}} & \cdots & e^{\xi_{N} \partial_{\varepsilon_{s}}} & \varphi\left(\lambda_{N}, \nu_{s+1}\right) & \cdots & \varphi\left(\lambda_{N}, \nu_{N}\right)
\end{array}\right| \times \\
& \times\left.\prod_{j=1}^{s} v_{r_{j}}\left(\lambda+\varepsilon_{j}\right) \prod_{1 \leqslant j<k \leqslant s} \frac{1}{e\left(\lambda+\varepsilon_{j}, \lambda+\varepsilon_{k}\right)}\right|_{\varepsilon_{1}=\cdots=\varepsilon_{s}=0} .
\end{aligned}
$$

Подчеркнем, что это выражение справедливо для неоднородной модели.

Рассмотрим теперь однородный предел. Будем рассматривать параметр $\lambda$ как параметр весов однородной модели, так что параметры $\xi_{1}, \ldots, \xi_{N}$ и $\nu_{s+1}, \ldots, \nu_{N}$ должны быть устремлены к нулю в этом пределе. Процедура взятия предела может быть выполнена аналогично работе [4], и она детально изложена в работе [11]. В результате мы получим

$$
\begin{aligned}
& Z_{r_{1}, \ldots, r_{s}}^{\text {bot }}=\frac{(a b)^{N(N-s)}}{\prod_{j=1}^{N-s-1} j ! \prod_{k=1}^{N-1} k !}\left|\begin{array}{cccccc}
\varphi(\lambda) & \cdots & \partial_{\lambda}^{N-s-1} \varphi(\lambda) & 1 & \cdots & 1 \\
\partial_{\lambda} \varphi(\lambda) & \cdots & \partial_{\lambda}^{N-s} \varphi(\lambda) & \partial_{\varepsilon_{1}} & \cdots & \partial_{\varepsilon_{s}} \\
\vdots & \ddots & \vdots & \vdots & \ddots & \vdots \\
\partial_{\lambda}^{N-1} \varphi(\lambda) & \cdots & \partial_{\lambda}^{2 N-s-2} \varphi(\lambda) & \partial_{\varepsilon_{1}}^{N-1} & \ldots & \partial_{\varepsilon_{s}}^{N-1}
\end{array}\right| \times \\
& \times\left.\prod_{j=1}^{s} \frac{\left(\sin \varepsilon_{j}\right)^{N-r_{j}}\left[\sin \left(\varepsilon_{j}-2 \eta\right)\right]^{r_{j}-1}}{\left[\sin \left(\varepsilon_{j}+\lambda-\eta\right)\right]^{N-s}} \prod_{1 \leqslant j<k \leqslant s} \frac{1}{\sin \left(\varepsilon_{j}-\varepsilon_{k}+2 \eta\right)}\right|_{\varepsilon_{1}=\cdots=\varepsilon_{s}=0}
\end{aligned}
$$

где в записи определителя мы изменили порядок столбцов по сравнению с формулой (4.6).

Для того чтобы выразить (4.7) через многократный интеграл, мы сначала преобразуем детерминантное $(N \times N)$-представление $(4.7)$ в детерминантное $(s \times s)$-представление, задаваемое в терминах некоторого набора ортогональных полиномов. Конструкция основана на следующих общих фактах из теории ортогональных полиномов (см., например, монографию [20]). Пусть $\left\{P_{n}(x)\right\}_{n=0}^{\infty}$ является набором ортогональных полиномов

$$
\int P_{n}(x) P_{m}(x) \mu(x) d x=h_{n} \delta_{n m},
$$


где областью интегрирования считается вещественная ось, и мы выберем нормировку так, что $P_{n}(x)=x^{n}+\cdots$. Пусть также $c_{n}$ обозначает $n$-й момент веса $\mu(x)$ :

$$
c_{n}=\int x^{n} \mu(x) d x, \quad n=0,1, \ldots
$$

Тогда $\operatorname{det}_{1 \leqslant j, k \leqslant N}\left\{c_{j+k-2}\right\}=h_{0} h_{1} \cdots h_{N-1}$ и, более общо, для $s=1, \ldots, N$ справедлива следующая формула:

$$
\begin{array}{cccccccc}
c_{0} & c_{1} & \cdots & c_{N-s-1} & 1 & 1 & \cdots & 1 \\
c_{1} & c_{2} & \cdots & c_{N-s} & x_{1} & x_{2} & \cdots & x_{s} \\
\vdots & \vdots & \ddots & \vdots & \vdots & \vdots & \ddots & \vdots \\
c_{N-1} & c_{N} & \cdots & c_{2 N-s-2} & x_{1}^{N-1} & x_{2}^{N-1} & \cdots & x_{s}^{N-1}
\end{array} \mid=
$$

$\mathrm{B}$ нашем случае $c_{n}:=\partial_{\lambda}^{n} \varphi(\lambda)$, и мера интегрирования $\mu(x) d x$ может быть найдена через преобразование Лапласа для функции $\varphi(\lambda)$; явные выражения приведены в работе [21].

Как и в работе [11], мы обозначим

$$
K_{n}(x)=\frac{n ! \varphi^{n+1}}{h_{n}} P_{n}(x),
$$

где $\varphi:=\varphi(\lambda)$, а $h_{n}$ то же, что и в формуле (4.8). Мы также введем функции

$$
\omega(\varepsilon):=\frac{a}{b} \frac{\sin \varepsilon}{\sin (\varepsilon-2 \eta)}, \quad \widetilde{\omega}(\varepsilon):=\frac{b}{a} \frac{\sin \varepsilon}{\sin (\varepsilon+2 \eta)},
$$

которые, в частности, удовлетворяют соотношению

$$
\frac{b}{c} \frac{\sin (\varepsilon-2 \eta)}{\sin (\varepsilon+\lambda-\eta)}=\frac{1}{\omega(\varepsilon)-1},
$$

где использованы краткие обозначения для весов $a:=\sin (\lambda+\eta), b:=\sin (\lambda-\eta)$ и $c:=\sin 2 \eta$. Принимая во внимание, что $\varphi=c / a b$, и используя соотношение

$$
\frac{\sin \left(\varepsilon_{1}+\lambda+\eta\right) \sin \left(\varepsilon_{2}+\lambda-\eta\right)}{\sin \left(\varepsilon_{1}-\varepsilon_{2}+2 \eta\right)}=\frac{1}{\varphi} \frac{\left(1-\widetilde{\omega}\left(\varepsilon_{1}\right)\right)\left(\omega\left(\varepsilon_{2}\right)-1\right)}{\widetilde{\omega}\left(\varepsilon_{1}\right) \omega\left(\varepsilon_{2}\right)-1},
$$

после применения формулы (4.10) к выражению (4.7) получим

$$
\begin{aligned}
Z_{r_{1}, \ldots, r_{s}}^{\text {bot }}= & \frac{Z_{N}}{a^{s(2 N-s+1) / 2} b^{s(s-3) / 2} c^{s}}\left(\frac{a}{b}\right)^{r_{1}+\cdots+r_{s}} \operatorname{det}_{1 \leqslant j, k \leqslant s}\left\{K_{N-s+j-1}\left(\partial_{\varepsilon_{k}}\right)\right\} \times \\
& \times\left.\prod_{j=1}^{s} \frac{\left[\omega\left(\varepsilon_{j}\right)\right]^{N-r_{j}-s+j}\left[\widetilde{\omega}\left(\varepsilon_{j}\right)\right]^{s-j}}{\left[\omega\left(\varepsilon_{j}\right)-1\right]^{N-s}} \prod_{1 \leqslant j<k \leqslant s} \frac{1}{\widetilde{\omega}\left(\varepsilon_{j}\right) \omega\left(\varepsilon_{k}\right)-1}\right|_{\varepsilon_{1}=\cdots=\varepsilon_{s}=0} .
\end{aligned}
$$


В выводе этой формулы мы также использовали формулу (2.18), чтобы выразить подходящий множитель через статистическую сумму $Z_{N}$.

Теперь мы готовы записать представление (4.15) как многократный интеграл. Будем следовать процедуре, развитой в работе [11]. Ключевым соотношением, справедливым для произвольной функции $f(z)$, регулярной в окрестности нуля, здесь является следующее:

$$
\left.K_{N-1}\left(\partial_{\varepsilon}\right) f(\omega(\varepsilon))\right|_{\varepsilon=0}=\frac{1}{2 \pi i} \oint_{C_{0}} \frac{(z-1)^{N-1}}{z^{N}} h_{N}(z) f(z) d z .
$$

Здесь $C_{0}$, как и выше, малый простой замкнутый положительно ориентированный контур, обходящий точку $z=0$, a $h_{N}(z)$ (не следует путать с $h_{n}$ в формуле (4.8)) является производящей функцией для одноточечной корреляционной функции, $h_{N}(z)=\sum_{r=1}^{N} H_{N}^{(r)} z^{r-1}$, где

$$
H_{N}^{(r)}=\left.K_{N-1}\left(\partial_{\varepsilon}\right) \frac{[\omega(\varepsilon)]^{N-r}}{[\omega(\varepsilon)-1]^{N-1}}\right|_{\varepsilon=0} .
$$

Эта функция может рассматриваться как случай $s=1$ для вероятности конфигурации ряда, $H_{N}^{(r)}:=H_{N, 1}^{(r)}$. Действительно, в этом случае статистическая сумма верхней подрешетки есть $Z_{r}^{\text {top }}=a^{N-r} b^{r-1} c$, a $Z_{r}^{\text {bot }}$ может быть получена из формулы (4.15), таким образом воспроизводя выражение (4.17).

Чтобы выписать результирующее представление многократным интегралом для $Z_{r_{1}, \ldots, r_{s}}^{\text {bot }}$, введем функции

$$
h_{N, s}\left(z_{1}, \ldots, z_{s}\right)=\frac{\operatorname{det}_{1 \leqslant j, k \leqslant s}\left\{z_{k}^{s-j}\left(z_{k}-1\right)^{j-1} h_{N-s+j}\left(z_{k}\right)\right\}}{\prod_{1 \leqslant j<k \leqslant s}\left(z_{k}-z_{j}\right)},
$$

которые могут рассматриваться как обобщение функции $h_{N}(z)$ на случай многих переменных (детальное обсуждение их свойств см. в работе [11]). Замечая, что

$$
\widetilde{\omega}(\varepsilon)=\frac{t^{2} \omega(\varepsilon)}{2 \Delta t \omega(\varepsilon)-1}
$$

где $t=b / a$ и $\Delta=\left(a^{2}+b^{2}-c^{2}\right) / 2 a b$, мы можем немедленно переписать представление в терминах ортогональных полиномов (4.15) с помощью формулы (4.16) следующим образом:

$$
\begin{aligned}
Z_{r_{1}, \ldots, r_{s}}^{\mathrm{bot}}= & Z_{N} \frac{\prod_{j=1}^{s} t^{j-r_{j}}}{a^{s(N-1)} c^{s}} \oint_{C_{0}} \cdots \oint_{C_{0}} \prod_{j=1}^{s} \frac{1}{z_{j}^{r_{j}}} \prod_{1 \leqslant j<k \leqslant s} \frac{z_{k}-z_{j}}{t^{2} z_{j} z_{k}-2 \Delta t z_{j}+1} \times \\
& \times h_{N, s}\left(z_{1}, \ldots, z_{s}\right) \frac{d^{s} z}{(2 \pi i)^{s}} .
\end{aligned}
$$

Эта формула и есть желаемое представление для $Z_{r_{1}, \ldots, r_{s}}^{\text {bot }}$, справедливое для однородной модели. 


\section{5. ВЕРОЯТНОСТЬ ОБРАЗОВАНИЯ ПУСТОТЫ}

Важным примером корреляционной функции, которая может быть построена из вероятности конфигурации ряда, является вероятность образования пустоты. Как и в работе [11], будем обозначать через $F_{N}^{(r, s)}$ вероятность того, что стрелки на первых $s$ горизонтальных ребрах (нумеруемых, как обычно, сверху), лежащих между $r$-й и $(r+1)$-й вертикальными прямыми (нумеруемыми, как обычно, справа) все направлены влево. Эквивалентно в силу граничных условий доменной стенки и правила льда мы можем определить эту величину как вероятность того, что последние $N-r$ вертикальных стрелок, расположенные между $s$-й и $(s+1)$-й горизонтальными прямыми, все направлены вниз и, следовательно (см. также рис. 2), имеет место соотношение

$$
F_{N}^{(r, s)}=\sum_{1 \leqslant r_{1}<r_{2}<\cdots<r_{s} \leqslant r} H_{N, s}^{\left(r_{1}, \ldots, r_{s}\right)} .
$$

Нашей целью здесь будет исследование выполнения этого суммирования для вероятности конфигурации ряда, даваемой формулами (2.19), (3.6) и (4.20), чтобы воспроизвести представления многократными интегралами для вероятности образования пустоты, полученные в работе [11].

Напомним вначале результаты работы [11]. Были получены следующие два представления многократными интегралами:

$$
\begin{aligned}
F_{N}^{(r, s)}= & (-1)^{s} \oint_{C_{0}} \ldots \oint_{C_{0}} \prod_{j=1}^{s} \frac{\left[\left(t^{2}-2 \Delta t\right) z_{j}+1\right]^{s-j}}{z_{j}^{r}\left(z_{j}-1\right)^{s-j+1}} \prod_{1 \leqslant j<k \leqslant s} \frac{z_{j}-z_{k}}{t^{2} z_{j} z_{k}-2 \Delta t z_{j}+1} \times \\
& \times h_{N, s}\left(z_{1}, \ldots, z_{s}\right) \frac{d^{s} z}{(2 \pi i)^{s}}= \\
= & \frac{(-1)^{s} Z_{s}}{s ! a^{s(s-1)} c^{s}} \oint_{C_{0}} \ldots \oint_{C_{0}} \prod_{j=1}^{s} \frac{\left[\left(t^{2}-2 \Delta t\right) z_{j}+1\right]^{s-1}}{z_{j}^{r}\left(z_{j}-1\right)^{s}} \prod_{\substack{j, k=1 \\
j \neq k}}^{s} \frac{z_{k}-z_{j}}{t^{2} z_{j} z_{k}-2 \Delta t z_{j}+1} \times \\
& \times h_{N, s}\left(z_{1}, \ldots, z_{s}\right) h_{s, s}\left(u\left(z_{1}\right), \ldots, u\left(z_{s}\right)\right) \frac{d^{s} z}{(2 \pi i)^{s}},
\end{aligned}
$$

где

$$
u(z):=-\frac{z-1}{\left(t^{2}-2 \Delta t\right) z+1} .
$$

Два представления в формуле (5.2) связаны симметризацией подынтегрального выражения в силу тождества

$$
\begin{aligned}
& \operatorname{Asym}_{z_{1}, \ldots, z_{s}}\left[\prod_{1 \leqslant j<k \leqslant s} \frac{\left[\left(t^{2}-2 \Delta t\right) z_{j}+1\right]\left(t^{2} z_{j} z_{k}-2 \Delta t z_{k}+1\right)}{\left(z_{j}-1\right)}\right]= \\
& \quad=\frac{Z_{s}}{s ! a^{s(s-1)} c^{s}} \prod_{j=1}^{s} \frac{\left[\left(t^{2}-2 \Delta t\right) z_{j}+1\right]^{s-1}}{\left(z_{j}-1\right)^{s-1}} \prod_{1 \leqslant j<k \leqslant s}\left(z_{k}-z_{j}\right) h_{s, s}\left(u\left(z_{1}\right), \ldots, u\left(z_{s}\right)\right) .
\end{aligned}
$$

Здесь $\operatorname{Asym}_{z_{1}, \ldots, z_{s}} f\left(z_{1}, \ldots, z_{s}\right):=(1 / s !) \sum_{P}(-1)^{[P]} f\left(z_{P_{1}}, \ldots, z_{P_{s}}\right)$, и сумма выполняется по перестановкам $P: 1, \ldots, s \mapsto P_{1}, \ldots, P_{s}$, где $[P]$ обозначает четность $P$. Подробное обсуждение доказательства тождества (5.4) дано в работе [11]. 
Обсудим теперь представление для вероятности образования пустоты, получаемое из формул $(2.19),(3.6)$ и (4.20) согласно соотношению (5.1). Прямая подстановка дает

$$
\begin{aligned}
F_{N}^{(r, s)}= & \oint_{C_{1}} \cdots \oint_{C_{1}} \frac{d^{s} w}{(2 \pi i)^{s}} \oint_{C_{0}} \cdots \oint_{C_{0}} \prod_{j=1}^{s} \frac{1}{\left(w_{j}-1\right)^{s}} \sum_{1 \leqslant r_{1}<r_{2}<\cdots<r_{s} \leqslant r} \prod_{j=1}^{s} \frac{w_{j}^{r_{j}-1}}{z_{j}^{r_{j}}} \times \\
& \times \prod_{1 \leqslant j<k \leqslant s} \frac{\left(w_{j}-w_{k}\right)\left(t^{2} w_{j} w_{k}-2 \Delta t w_{j}+1\right)\left(z_{k}-z_{j}\right)}{t^{2} z_{j} z_{k}-2 \Delta t z_{j}+1} h_{N, s}\left(z_{1}, \ldots, z_{s}\right) \frac{d^{s} z}{(2 \pi i)^{s}}
\end{aligned}
$$

так что, вычисляя многократную сумму и интегрируя по одному из наборов переменных (например, по $w_{1}, \ldots, w_{s}$ ), мы должны воспроизвести с точностью до симметризации подынтегрального выражения $s$-кратное интегральное представление (5.2).

Чтобы исследовать эту задачу, рассмотрим вначале многократную сумму в формуле (5.5). Замечая, что интеграл по переменной $z_{j}$ равен нулю, если $r_{j} \leqslant 0$, поскольку в этом случае подынтегральное выражение регулярно в точке $z_{j}=0$, мы можем в формуле (5.5) заменить сумму по значениям $1 \leqslant r_{1}<r_{2}<\cdots<r_{s} \leqslant r$ суммой по значениям $-\infty<r_{1}<r_{2}<\cdots<r_{s} \leqslant r$. Тогда, обозначая $X_{j}=z_{j} / w_{j}$, суммирование может быть выполнено с помощью тождества

$$
\sum_{-\infty<r_{1}<r_{2}<\cdots<r_{s} \leqslant r} \prod_{j=1}^{s} \frac{1}{X_{j}^{r_{j}}}=\prod_{j=1}^{s} \frac{1}{X_{j}^{r-s+j}\left(1-\prod_{l=1}^{j} X_{l}\right)}
$$

которое легко проверить разложением знаменателей в правой части в ряды Тейлора. В результате получим, что формула (5.5) сводится к выражению

$$
\begin{aligned}
F_{N}^{(r, s)}= & \oint_{C_{1}} \cdots \oint_{C_{1}} \frac{d^{s} w}{(2 \pi i)^{s}} \oint_{C_{0}} \cdots \oint_{C_{0}} \prod_{j=1}^{s} \frac{w_{j}^{r}}{\left(w_{j}-1\right)^{s} z_{j}^{r-s+j}\left(\prod_{l=1}^{j} w_{l}-\prod_{l=1}^{j} z_{l}\right)} \times \\
& \times \prod_{1 \leqslant j<k \leqslant s} \frac{\left(w_{j}-w_{k}\right)\left(t^{2} w_{j} w_{k}-2 \Delta t w_{j}+1\right)\left(z_{k}-z_{j}\right)}{t^{2} z_{j} z_{k}-2 \Delta t z_{j}+1} h_{N, s}\left(z_{1}, \ldots, z_{s}\right) \frac{d^{s} z}{(2 \pi i)^{s}}
\end{aligned}
$$

и нам осталось выполнить $s$-кратное интегрирование.

Будем выполнять интегрирование по переменным $w_{1}, \ldots, w_{s}$ в выражении (5.7). Для этого рассмотрим эквивалентный интеграл, у которого подынтегральное выражение полностью симметрично относительно перестановок этих переменных. Определим функцию

$$
\begin{aligned}
\Phi_{s}\left(w_{1}, \ldots, w_{s} ; z_{1}, \ldots, z_{s}\right)= & \prod_{1 \leqslant j<k \leqslant s} \frac{1}{w_{k}-w_{j}} \times \\
& \times \operatorname{Asym}_{w_{1}, \ldots, w_{s}}\left[\frac{\prod_{1 \leqslant j<k \leqslant s}\left(t^{2} w_{j} w_{k}-2 \Delta t w_{j}+1\right)}{\prod_{j=1}^{s}\left(\prod_{l=1}^{j} w_{l}-\prod_{l=1}^{j} z_{l}\right)}\right] .
\end{aligned}
$$


Интегрирование по переменным $w_{1}, \ldots, w_{s}$ приводит к результату

$$
\begin{aligned}
\oint_{C_{1}} & \cdots \oint_{C_{1}} \prod_{j=1}^{s} \frac{w_{j}^{r}}{\left(w_{j}-1\right)^{s}} \prod_{1 \leqslant j<k \leqslant s}\left(w_{j}-w_{k}\right)^{2} \Phi_{s}\left(w_{1}, \ldots, w_{s} ; z_{1}, \ldots, z_{s}\right) \frac{d^{s} w}{(2 \pi i)^{s}}= \\
& =(-1)^{s(s-1) / 2} s ! \Phi_{s}\left(1, \ldots, 1 ; z_{1}, \ldots, z_{s}\right)
\end{aligned}
$$

который может быть легко получен, если заметить, что при вычислении вычетов достаточно дифференцировать только множитель $\prod_{j<k}\left(w_{j}-w_{k}\right)^{2}$.

Окончательно желаемый результат для вероятности образования пустоты сводится к доказательству тождества

$$
\begin{aligned}
& s ! \operatorname{Asym}_{z_{1}, \ldots, z_{s}}\left[\Phi_{s}\left(1, \ldots, 1 ; z_{1}, \ldots, z_{s}\right) \prod_{1 \leqslant j<k \leqslant s}\left[z_{j}\left(t^{2} z_{j} z_{k}-2 \Delta t z_{k}+1\right)\right]\right]= \\
& =\frac{(-1)^{s(s+1) / 2}}{\prod_{j=1}^{s}\left(z_{j}-1\right)} \operatorname{Asym}_{z_{1}, \ldots, z_{s}}\left[\prod_{1 \leqslant j<k \leqslant s} \frac{\left[\left(t^{2}-2 \Delta t\right) z_{j}+1\right]\left(t^{2} z_{j} z_{k}-2 \Delta t z_{k}+1\right)}{\left(z_{j}-1\right)}\right] .
\end{aligned}
$$

Это тождество должно быть использовано совместно с тождеством (5.4), чтобы воспроизвести формулу (5.2). Оказывается, что тождество (5.10) весьма трудно доказать непосредственно, и в настоящее время нам удалось лишь проверить его с помощью компьютерных вычислений для небольших значений $s$. Отметим, что весьма похожие тождества обсуждались в работах [22]-[24].

В заключение в настоящей работе мы ввели и вычислили нелокальную корреляционную функцию шестивершинной модели с граничными условиями доменной стенки - вероятность конфигурации ряда. Она дается в виде произведения двух сомножителей, которые могут быть рассмотрены как статистические суммы на верхней и нижней подрешетках начальной решетки (см. рис. 2). Мы выразили эти статистические суммы через многократные интегралы (см. формулы (3.6) и (4.20)). Вероятность конфигурации ряда может быть использована для вычисления других корреляционных функций при условии, что могут быть выполнены суммирования, аналогичные суммированию, присутствующему в формуле (5.1). Чтобы проиллюстрировать это, мы рассмотрели задачу воспроизведения известного результата для вероятности образования пустоты. Мы показали, что в этом случае задача сводится к тождеству (5.10). Прямое доказательство этого тождества в дополнение к непрямому, следующему из известного равенства между (5.2) и (5.5), может оказаться полезным для вычисления других корреляционных функций.

Благодарности. Авторы признательны Н. М. Боголюбову, Д. Ромику и П. ЗиннЖюстену за полезные обсуждения. Ф. Коломо благодарит за финансовую поддержку MIUR, грант PRIN 2007JHLPEZ, и программу INSTANS от Европейского Научного фонда. А.Г. Пронько благодарит фонд Александра фон Гумбольдта за финансовую поддержку во время его работы в университете г. Вупперталь, Германия, а также РФФИ (грант № 10-01-00600) и программу РАН "Математические методы нелинейной динамики" за частичную поддержку. 
Ф. КОЛОмо, А. г. ПРОНЬКО

\section{Список литературы}

[1] V.E. Korepin, N. M. Bogoliubov, A. G. Izergin, Quantum Inverse Scattering Method and Correlation Functions, Cambridge Univ. Press, Cambridge, 1993.

[2] V.E. Korepin, Commun. Math. Phys., 86:3 (1982), 391-418.

[3] А. Г. Изергин, Докл. АН СССР, 297:2 (1987), 331-334.

[4] A. G. Izergin, D. A. Coker, V. E. Korepin, J. Phys. A, 25:16 (1992), 4315-4334.

[5] F. Colomo, A. G. Pronko, SIAM J. Discrete Math., 24:4 (2010), 1558-1571, arXiv: 0803.2697.

[6] F. Colomo, A. G. Pronko, J. Stat. Phys., 138:4-6 (2010), 662-700, arXiv: 0907.1264.

[7] N. M. Bogoliubov, A. V. Kitaev, M. B. Zvonarev, Phys. Rev. E, 65:2 (2002), 026126, 4 pp., arXiv: cond-mat/0107146.

[8] N. M. Bogoliubov, A. G. Pronko, M. B. Zvonarev, J. Phys. A, 35:27 (2002), 5525-5541, arXiv: math-ph/0203025.

[9] O. Foda, I. Preston, J. Stat. Mech. Theory Exp., 11 (2004), P11001, 24 pp., arXiv: math-ph/0409067.

[10] F. Colomo, A. G. Pronko, J. Stat. Mech. Theory Exp., 5 (2005), 05010, 21 pp., arXiv: math-ph/0503049.

[11] F. Colomo, A. G. Pronko, Nucl. Phys. B, 798:3 (2008), 340-362, arXiv: 0712.1524.

[12] K. Motegi, Physica A, 390:20 (2011), 3337-3347, arXiv: 1101.0187.

[13] P. Di Francesco, N. Reshetikhin, Commun. Math. Phys., 309:1 (2012), 87-121, arXiv: 0908.1630.

[14] I. Fischer, D. Romik, Adv. Math., 222:6 (2009), 2004-2035, arXiv: 0903.5073.

[15] Л. А. Тахтаджян, Л. Д. Фаддеев, УМН, 34:5(209) (1979), 13-63.

[16] A. G. Izergin, V. E. Korepin, N. Yu. Reshetikhin, J. Phys. A, 20:14 (1987), 4799-4822.

[17] R. J. Baxter, Exactly Solved Models in Statistical Mechanics, Academic Press, London, 1982.

[18] N. Kitanine, J.-M. Maillet, V. Terras, Nucl. Phys. B, 554:3 (1999), 647-678, arXiv: math-ph/9907020.

[19] L. Fischer, Adv. Appl. Math., 37:2 (2006), 249-267, arXiv: math/0501102.

[20] G. Szegö, Orthogonal Polinomials, Colloquium Publications, v. XXIII, 4th ed., AMS, Providence, RI, 1975.

[21] P. Zinn-Justin, Phys. Rev. E, 62:3 (2000), 3411-3418, arXiv: math-ph/0005008.

[22] П. Зинн-Жюстен, Ф. Ди Франческо, ТМФ, 154:3 (2008), 387-408.

[23] D. Zeilberger, Proof of a conjecture of Philippe Di Francesco and Paul Zinn-Justin related to the $q K Z$ equation and to Dave Robbins' two favorite combinatorial objects, 2007, http://www.math.rutgers.edu/ zeilberg/mamarim/mamarimhtml/diFrancesco.html.

[24] C. A. Tracy, H. Widom, Commun. Math Phys., 279:3 (2008), 815-844; Erratum, 304:3 (2011), 875-878, arXiv: 0704.2633. 